\title{
Mesenchymal Stem Cells As A Therapeutic Option For Patients With ALS
}

Dariusz Boruczkowski ${ }^{1}$, Dominika Gładysz ${ }^{1}$, Mariusz Grudniak ${ }^{1}$, Karol Kosterna ${ }^{1}$ and Katarzyna Pawelec ${ }^{1,2^{*}}$

${ }^{1}$ Polish Stem Cells Bank, Warsaw, Poland

${ }^{2}$ Department of Pediatrics, Hematology and Oncology, Medical University of Warsaw, Poland

\begin{abstract}
It has been postulated that mesenchymal stem cells could play a role in treatment of patients with amyotrophic lateral sclerosis. Variety of mechanism behind MSC action has been proposed including immunomodulation and delivery of trophic factors. The scientific rationale in preclinical studies is being proved along with first clinical uses. Recent advances in stem cell research rises new hope for patients with ALS.
\end{abstract}

Keywords: Mesenchymal stem vells; Amyotrophic lateral sclerosis; Pathophysiology

\section{Background}

Amyotrophic lateral sclerosis (ALS) is a progressive neurodegenerative disease with poorly understood pathophysiology. Death of upper and lower motor neuron results in weakness and paralysis of skeletal muscles including respiratory musculature. Disease typically begins with muscle weakness and atrophy or progressive bulbar palsy and leads to respiratory distress and death about 5 years from the diagnosis. About 1-2 new cases per 100000 per year are diagnosed, about $10 \%$ is familial and connected with superoxide dismutase 1 (SOD1) mutation.

Despite scientific development, there is no successful treatment for ALS, riluzol only prolongs survival for about $10-20 \%$ [1]. Stem cell treatment might be a disease-modifying strategy for ALS patients. Mesenchymal stem cells (MSC) have been defined by International Society for Cellular Therapy, they are known to be weakly or nonimmunogenic and thus applicable in an allogeneic setting [2]. Wharton's jelly-derived MSC (WJ-MSC) seem to be a preferable source because of easiness and safety of the harvesting procedure as well as a rich number of cells contained in the umbilical cord. WJ-MSC have high immunomodulatory capabilities as well as good proliferation and differentiation potential. Their characteristics close to embryo-derived stem cells but with lower risk of potential tumorigenesis [3].

\section{Mechanism of action}

The mechanism behind ALS are not entirely known. Studies on animal models have been undertaken to deepen understanding of the disease and explore potential mechanisms of treatment with MSC. The hypothesis include indirect effects such as the delivery of growth factors in situ, modulation of glial cells secretome, up-regulating regulatory T-cell activity. Preclinical studies showed that MSC express 12 neural genes and 11 transcription factors, and are able to differentiate into neural and glial cells in an appropriate media [4].

Sun et al. [5] study on ALS animal model proved that MSC plays a role in neuroprotection of motor neurons, astrocytes, microglia, and modulates their response to apoptosis. Modulation of response to inflammation by down-regulating expression of TNFa and IL-6 and iNOS was observed as well. According to Uccelli et al. [6] study, paracrine mechanisms are more probable than local production of growth factors since MSC intravenous infusion into mouse ALS model lead to amelioration of motor neuron function, slowdown of weight loss and survival improvement, despite low engraftment rates. The results of co-culture of MSC with peripheral blood mononuclear cells derived from ALS patients, showed up-regulation of anti-inflammatory cytokines levels including IL-4, IL-10 and TGF-b, and increased regulatory T-cell/total lymphocyte ratio [7].

\section{Preclinical studies}

It has been confirmed during ex vivo experiment that WJ-MSC maintain their immunomodulatory properties upon a long-term culture and preserve genetic stability through expansion up to 15 passages without chromosomal changes and malignant transformation both in vitro and after MSC injection into nude mice with follow-up of 4 weeks [8]. Some investigators in preclinical studies gave insight into possible MSC mechanisms in treating ALS. Marconi et al. [9] study explored effect of systemic injection of autologous adipose tissue-derived MSC into mouse model of ALS. Stem cell administration slowed down the deterioration of motor neuron function by $4-6$ weeks. The study revealed higher number of lumbar motor neurons on the neuropathologic examination as well as higher level of both glial-derived neurotrophic (GDFN) and basic fibroblast growth factors (bFGF). Since MSC are able to produce bFGF, but not GDNF, the investigators hypothesize that MSC in ALS treatment acts through enhancement of neuroprotection by producing soluble factors that modulate biological functions of local glial cells. Boido et al. [10] administered human MSC into cisterna lumbalis of ALS mouse model and observed prevention of IL-10 and vascular endothelial growth factor down-regulation and increased IL13 production. Another study showed promising results after multiple intravenous infusions of amniotic-derived MSC into ALS mouse model [11]. An intravenous delivery of bone marrow-derived MSC (BM-MSC) on animal model was found to be effective in ameliorating disease course and alternating cytokine profile [12].

\section{Clinical setting}

The first use of MSC in a clinical setting of ALS was reported by Mazzini et al. [13]. The study was done on 9 patients who received

*Corresponding author: Katarzyna Pawelec, Department of Pediatrics, Hematology and Oncology Medical University of Warsaw, Zwirki I Wigury 63A 02-091 Warszawa, Poland, Tel: +4822 2231796 21; Fax: +4822 2231796 21; E-mail: katarzyna.pawelec@litewska.edu.pl

Received March 9, 2016 Accepted April 21, 2016 Published April 27, 2016

Citation: Boruczkowski D, Gładysz D, Grudniak M, Kosterna K, Pawelec K (2016) Mesenchymal Stem Cells As A Therapeutic Option For Patients With ALS. Gen Med (Los Angel) 4: 235. doi:10.4172/2327-5146.1000235

Copyright: (c) 2016 Boruczkowski D, et al. This is an open-access article distributed under the terms of the Creative Commons Attribution License, which permits unrestricted use, distribution, and reproduction in any medium, provided the original author and source are credited. 
autologous BM-MSC via intra spinal delivery in an unstandardized dose and number of injections. There were no serious adverse effects and no clinical improvement. Four patients complied of pain in the intercostal area, 5 patients reported dysesthesia in lower limbs. Symptoms subsided up to 6 weeks after MSC administration. The investigators concluded that MSC administration in ALS patients appears to be safe and well-tolerated [13]. The same patients were evaluated 3 years after MSC therapy. A slowdown of the decline of the forced vital capacity was observed in 4 patients [14]. The same therapeutic approach was repeated on 10 patients with ALS. Similar adverse effects were described [15]. The long-term follow up of above-described patients equaled nearly 9 years. No slowing down of ALS clinical course was observed, however no disease acceleration or new tissue formation was reported [16].

A study conducted by Prabhakar et al. [17] evaluated influence of autologous BM-MSC over ALS disease course. Ten patients underwent lumbar puncture with stem cell administration and no adverse effects were observed. The authors concluded that MSC administration led to disease stabilization in 1 year period of follow-up since no significant deterioration in ALS Functional Rating Scale was noted.

Karussis et al. [18] intravenously or intrathecally infused autologous BM-MSC in 19 ALS patients, in a few cases stem cells were labelled with superparamagnetic iron oxides. Adverse effects were non-serious and self-limiting, and included: fever, headache, lower limb pain, dyspnea. No late adverse effects were noted during follow-up of 6-25 months. The course of the disease remained stable or lightly improved in most of the cases. MRI study on patients who received labelled cells showed that MSC infused in the lumbar area were detected in the occipital horns of the ventricles, subarachnoid space and spinal cord. Immunological analysis proved that first immunomodulatory effects were detectable just 4 hours after MSC administration and consisted of downregulation of lymphocyte proliferative responses and up-regulation of CD4+CD25+ regulatory T cells [18].

Another study administered autologous mesenchymal stem cells intravenously and intrathecally. Stem cells infused through lumbar puncture were committed to neuronal differentiation. Disease progression was observed in study and control group, however its dynamics was significantly slower in patients who received stem cell therapy [19].

Oh et al. [20] conducted an open label phase I clinical trial with intrathecal injections of autologous BM-MSC. Seven out of 8 patients received 2 doses with time interval of about 1 month. No serious adverse effects were noted within 12 months of follow-up. The course of the disease seemed to be slower after therapy in comparison to previous decline. A retrospective analysis of 57 ALS patients, including 20 controls and 37 patients treated with autologous bone marrow mononuclear cells, showed survival benefit of stem cell therapy [21]. Another approach was demonstrated by Deda et al. [22] and Blanquer et al. [23]. The first above-mentioned study delivered bone marrowderived hematopoietic progenitor cells were to 13 patients via total laminectomy and under general anesthesia [22]. The second one administered neurosurgically BM-MNC to patients with bulbar-onset ALS and noted slower disease progression [23].

\section{Summary}

Application of stem cells as an actual therapy for ALS patients is still in debate. It is a promising method, however current results are difficult to evaluate due to vast heterogeneity of patients and therapeutic schemes as well as lack of long-term follow-up. A growing number of clinical trials undertake this issue to assess answers about MSC efficacy and safety. WJ-MSC seem to be an interesting source for therapeutic use.

\section{References}

1. Sreedharan J, Brown RH Jr (2013) Amyotrophic lateral sclerosis: Problems and prospects. Ann Neurol 74: 309-316.

2. Dominici M, Le Blanc K, Mueller I, Slaper-Cortenbach I, Marini F, et al. (2006) Minimal criteria for defining multipotent mesenchymal stromal cells. The International Society for Cellular Therapy position statement. Cytotherapy 8 : 315-317.

3. Watson N, Divers R, Kedar R, Mehindru A, Mehindru A, et al. (2015) Discarded Wharton jelly of the human umbilical cord: a viable source for mesenchymal stromal cells. Cytotherapy 17: 18-24.

4. Blondheim NR, Levy YS, Ben-Zur T, Burshtein A, Cherlow T, et al. (2006) Human mesenchymal stem cells express neural genes, suggesting a neural predisposition. Stem Cells Dev 15: 141-164

5. Sun $H$, Bénardais $K$, Stanslowsky $N$, Thau-Habermann $N$, Hensel $N$, et al. (2013) Therapeutic potential of mesenchymal stromal cells and MSC conditioned medium in Amyotrophic Lateral Sclerosis (ALS)-in vitro evidence from primary motor neuron cultures, NSC-34 cells, astrocytes and microglia. PloS one 8: e72926.

6. Uccelli A, Milanese M, Principato MC, Morando S, Bonifacino T, et al. (2012) Intravenous mesenchymal stem cells improve survival and motor function in experimental amyotrophic lateral sclerosis. Molecular Medicine 18: 794-804.

7. Kwon MS, Noh MY, Oh KW, Cho KA, Kang BY, et al. (2014) The immunomodulatory effects of human mesenchymal stem cells on periphera blood mononuclear cells in ALS patients. Journal of Neurochemistry 131: 206218

8. Chen G, Yue A, Ruan Z, Yin Y, Wang R, et al. (2014) Human umbilical cordderived mesenchymal stem cells do not undergo malignant transformation during long-term culturing in serum-free medium. PLoS One 9: e98565.

9. Marconi S, Bonaconsa M, Scambi I, Squintani GM, Rui W, et al. (2013) Systemic treatment with adipose-derived mesenchymal stem cells ameliorates clinical and pathological features in the amyotrophic lateral sclerosis murine model. Neuroscience 248: 333-343.

10. Faravelli I, Riboldi G, Nizzardo M, Simone C, Zanetta C, et al. (2014) Stem cell transplantation for amyotrophic lateral sclerosis: therapeutic potential and perspectives on clinical translation. Cell and Molecular Life Science 71: 3257 268.

11. Sun $H$, Hou Z, Yang H, Meng M, Li P (2014) Multiple systemic transplantations of human amniotic mesenchymal stem cells exert therapeutic effects in an ALS mouse model. Cell and Tissue Research 357: 571-582.

12. Forostyak S, Homola A, Turnovcova K, Svitil P, Jendelova P, et al. (2014) Intrathecal delivery of mesenchymal stromal cells protects the structure of altered perineuronal nets in SOD1 rats and amends the course of ALS. Stem Cells 32: 3163-3172.

13. Mazzini L, Fagioli F, Boccaletti R, Mareschi K, Oliveri G, et al. (2003) Stem cell therapy in amyotrophic lateral sclerosis: a methodological approach in humans. Amyotrophic Lateral Sclerosis and Other Motor Neuron Disorders 4: 158-161.

14. Mazzini L, Mareschi K, Ferrero I, Vassallo E, Oliveri G, et al. (2006) Autologous mesenchymal stem cells: clinical applications in amyotrophic lateral sclerosis. Neurological Research 28: 523-526.

15. Mazzini L, Ferrero I, Luparello V, Rustichelli D, Gunetti M, et al. (2010) Mesenchymal stem cell transplantation in amyotrophic lateral sclerosis: Phase I clinical trial. Neurol 223: 229-237

16. Mazzini L, Mareschi K, Ferrero I, Miglioretti M, Stecco A, et al. (2012) Mesenchymal stromal cell transplantation in amyotrophic lateral sclerosis: a long-term safety study. Cytotherapy $14: 56-60$.

17. Prabhakar S, Marwaha N, Lal V, Sharma RR, Rajan R, et al. (2012) Autologous bone marrow-derived stem cells in amyotrophic lateral sclerosis: a pilot study. Neurol India 60: 465-469.

18. Karussis D, Karageorgiou C, Vaknin-Dembinsky A, Gowda-Kurkalli B, Gomor $\mathrm{JM}$, et al. (2010) Safety and immunological effects of mesenchymal stem cell transplantation in patients with multiple sclerosis and amyotrophic latera sclerosis. Archives of Neurology 67: 1187-1194. 
Citation: Boruczkowski D, Gładysz D, Grudniak M, Kosterna K, Pawelec K (2016) Mesenchymal Stem Cells As A Therapeutic Option For Patients With ALS. Gen Med (Los Angel) 4: 235. doi:10.4172/2327-5146.1000235

19. Rushkevich YN, Kosmacheva SM, Zabrodets GV, Ignatenko SI, Goncharova NV, et al. (2015) The use of autologous mesenchymal stem cells for cell therapy of patients with amyotrophic lateral sclerosis in belarus. Bulletin of Experimental Biology and Medicine 159: 576-581.

20. Oh KW, Moon C, Kim HY, Oh SI, Park J, et al. (2015) Phase I trial of repeated intrathecal autologous bone marrow-derived mesenchymal stromal cells in amyotrophic lateral sclerosis. Stem Cells Translational Medicine 4: 590-597.

21. Sharma AK, Sane HM, Paranjape AA, Gokulchandran N, Nagrajan A, et al. (2015) The effect of autologous bone marrow mononuclear cell transplantation on the survival duration in Amyotrophic Lateral Sclerosis - a retrospective controlled study. American Journal of Stem Cells 4: 50-65.

22. Deda H, Inci MC, Kürekçi AE, Sav A, Kayihan K, et al. (2009) Treatment of amyotrophic lateral sclerosis patients by autologous bone marrow-derived hematopoietic stem cell transplantation: a 1-year follow-up. Cytotherapy 11 18-25.

23. Blanquer M, Pérez Espejo MA, Iniesta F, Gómez Espuch J, Meca J, et al (2010) Bone marrow stem cell transplantation in amyotrophic lateral sclerosis: technical aspects and preliminary results from a clinical trial. Methods and Findings in Experimental and Clinical Pharmacology 32: 31-37. 